Cahiers de recherches médiévales

Journal of medieval studies

$4 \mid 1997$

Être père à la fin du Moyen Âge

\title{
Louis de Bruges, lecteur de Christine de Pizan
}

\section{Charity Cannon Willard}

\section{(2) OpenEdition}

Journals

Édition électronique

URL : https://journals.openedition.org/crm/955

DOI : $10.4000 / \mathrm{crm} .955$

ISSN : $1955-2424$

Éditeur

Honoré Champion

Édition imprimée

Date de publication : 15 décembre 1997

ISSN : 1272-9752

Référence électronique

Charity Cannon Willard, "Louis de Bruges, lecteur de Christine de Pizan », Cahiers de recherches médiévales [En ligne], 4 | 1997, mis en ligne le 15 janvier 2007, consulté le 15 décembre 2022. URL: http://journals.openedition.org/crm/955; DOI : https://doi.org/10.4000/crm.955

Ce document a été généré automatiquement le 15 décembre 2022.

Tous droits réservés 


\title{
Louis de Bruges, lecteur de Christine de Pizan $^{1}$
}

\author{
Charity Cannon Willard
}

1 Les manuscrits les plus anciens des œuvres de Christine de Pizan ont, bien entendu, un intérêt spécial; mais on apprend beaucoup d'une étude de ceux qui furent copiés plus tard, même vers la fin du quinzième siècle ou au début du seizième, car c'est ainsi que l'on peut connaître les lecteurs des œuvres de Christine aussi bien que les œuvres qui avaient le plus grand succès dans le temps.

2 Parmi les lecteurs, il vaut la peine de signaler Louis de Bruges (ou Lodewijk van Gruuthuse), qui était certainement parmi les plus intéressants. Cet homme célèbre est né à Bruges vers 1427; son titre de Seigneur de Gruuthuse vient d'un fief situé aux alentours de Bruges, ville dans laquelle on voit toujours son magnifique hôtel, à présent devenu musée. La bibliothèque impressionnante établie dans cet hôtel fut étudiée il y assez longtemps par Josèphe Van Praet, natif de Bruges et devenu bibliothécaire à la Bibliothèque Nationale de Paris. C'est en 1830 qu'il publia ses Recherches sur Louis de Bruges, avec une liste de 102 manuscrits ayant appartenue à ce Seigneur. Dans des catalogues plus récents on trouve encore dix-huit manuscrits. Assez récemment, comme suite à une magnifique exposition organisée à Bruges, Maximilin P.J. Martins a édité un volume intitulé Lodewijk van Gruuthyse, mecenas en Europees Diplomaat ca. 1427 1492 (Bruges, 1992) et comprenant des. résumés en français. Tout particulièrement, les illustrations des manuscrits sont magnifiques. La fin du volume comporte une liste des manuscrits identifiés comme ayant fait partie de la bibliothèque de Louis de Bruges. Si l'on s'intéresse surtout aux manuscrits de Christine, on trouve malheureusement dans cet ouvrage plusieurs erreurs d'importance. Je voudrais donc aujourd'hui noter ces erreurs avec l'espoir de les corriger.

3 En premier lieu, disons encore un mot sur la vie de Louis de Bruges, ce qui nous aidera à mieux comprendre l'importance de celui-ci comme bibliophile. Dans sa jeunesse, il se préparait à faire carrière à la cour des ducs de Bourgogne, y compris une carrière militaire. En 1452, le duc Philippe le Bon le nomma gouverneur de sa ville natale, et, l'année suivante, l'arma chevalier à la bataille de Gavère contre les Gantois en révolte. 
En 1445, Louis avait épousé Marguerite van Borsele, fille d'une famille très ancienne et distinguée (son père était amiral), et, en 1458, quand elle accoucha d'un fils, Philippe le Bon fut le parrain.

4 À partir de 1458, le duc confia régulièrement à Louis des missions diplomatiques, et c'est sans doute à la conclusion d'une d'entre elles qu'en 1461 il lui conféra l'ordre de la Toison d'Or. Vers la même époque, Louis devint officier (stadthouder) d'une région des territoires bourguignons (la Hollande, la Zélande et la Frise). Il est évident que ses contacts avec le duc de Bourgogne étaient constants et sans doute comprenaient des intérêts de bibliophile : sa bibliothèque en effet, visiblement inspirée de celle des ducs de Bourgogne, en fournit la preuve. À très peu d'exceptions près, aucun ouvrage ne figure dans la bibliothèque de Louis de Bruges qui ne se trouve, en un ou plusieurs exemplaires, dans la bibliothèque de Philippe le Bon. Ceci n'a d'ailleurs rien de très remarquable, car l'activité de bibliophile de Louis de Bruges semble commencer vers 1460. Il est aussi évident qu'en bien des cas, les deux hommes se servaient, pour leurs commandes respectives de manuscrits, des mêmes sources, des mêmes ateliers à Bruges ou à Gand.

5 Par ailleurs, il convient de noter l'intérêt particulier que le roi Édouard IV d'Angleterre porta à la bibliothèque de Louis. Ce dernier, en tant que stadthouder, se trouva en position de recevoir le roi parti en exil en lui offrant son hôtel de Bruges comme logement. Après son retour en Angleterre Édouard le récompensa en lui conférant le titre de «Earl of Winchester ».

6 Édouard fut certainement impressionné la bibliothèque qu'il trouva dans l'hôtel de Bruges. C'est ainsi que se trouvent à ce jour à la British Library 21 volumes copiés et enluminés à Bruges, pour la plupart des textes en français. Dans deux d'entre eux, les armoiries de Louis de Bruges ont même été remplacées par celles d'Édouard, indiquant peut-être des dons au roi. Ces manuscrits représentent la plus ancienne collection anglaise, conservée dans son ensemble, existant aujourd'hui.

7 Conséquence quelque peu inattendue de ce contact avec l'Angleterre, est la présence, dans la bibliothèque de Louis de Bruges - comme d'ailleurs dans celle de Philippe le Bon -, de quelques ouvrages de la Bibliothèque Royale de France, fondée par Charles V, mais dispersée par le duc de Bedford pendant sa régence, et passée en grande partie en Angleterre. Un manuscrit qui nous intéresse, mais qui date plutôt de l'époque de Charles VI, est la collection des œuvres poétiques offertes par Christine de Pizan à la reine, Isabeau de Bavière. Ce manuscrit partagea les fortunes de la Bibliothèque Royale, et il est à présent le célèbre Harley 4431 de la British Library.

8 L'histoire généralement acceptée de ce manuscrit indique que le duc de Bedford le donna à sa seconde femme, Jacquette de Luxembourg, dont on trouve la signature sur le premier feuillet de garde. Après la mort de Bedford, Jacquette se remaria avec Sir Richard Wydeville, comte Rivers. L'aînée des fils nés de ce mariage fut Anthony Wydeville dont l'autographe se trouve dans le manuscrit à côté de la signature de sa mère. C'est lui qui traduisit en anglais les Proverbes Moraux de Christine. C'était sans doute après la mort en disgrâce de ce dernier, en 1482, que le manuscrit de Christine passa à la bibliothèque de Louis de Bruges, qui a aussi écrit son nom, avec sa devise « plus est en vous » sur le même feuillet de garde.

9 L'étude éditée par Martens suggère une incertitude sur l'histoire de ce manuscrit, mais le nom et la devise sur le feuillet de garde semblerait laisser peu de doute. Il est vrai 
qu'on ne sait pas en quelles circonstances ce manuscrit est retourné en Angleterre après la mort de Louis de Bruges, car il n'y a plus de signature avant le dix-septième siècle, au moment où le duc de Newcastle a indiqué sa possession en 1676. Sa petite fille, Lady Henriette Cavendish Holles, épousa Edward Harley, devenu comte d'Oxford en 1724. C'est ainsi que le manuscrit entra, avec la collection Harley, dans les collectionsbritanniques en 1753.

D'après l'étude de Van Praet, on sait que la plus grande partie des manuscrits de la bibliothèque de Bruges sont passés, après la mort de Louis, dans la bibliothèque de Louis XII au Château de Blois. On sait aussi qu'en 1516 cette bibliothèque royale comptait 254 manuscrits, dont les 102 de Bruges constituait une partie importante. Il ne faut pas oublier de la mère de Louis, Marie d'Orléans, était la nièce de Philippe le Bon et avait passé sa jeunesse à la cour de Bourgogne. Elle aussi était bibliophile et connaissait sans doute, au moins de réputation, la bibliothèque de Louis de Bruges.

11 À la fin du livre de Martens, on trouve une liste détaillée de ces manuscrits, maintenant à Paris et ailleurs. On s'étonne d'y trouver mentionné un exemplaire de Mélibée et Prudence attribué à Christine (B.N.Ms.fr.1090). On sait depuis quelque temps que cette attribution est fausse. Le texte est plutôt une traduction du Liber consolationis et Consilii d'Albertano da Brescia faite par Renaud de Louhans. Ce texte, la source du Tale of Melibee de Chaucer, fut édité par J. Burke Severs avant 1941. (Sources and Analogues of Chaucer's Canterbury Tales, ed. W.F. Bryant et Germaine Dempster, Univ. of Chicago Press, 1941, pp. 560-614). Plus récemment, une thèse de doctorat a examiné l'influence de Renaud de Louhans sur le Livre de Prudence de Christine. (Angus A. Graham, "The German 'Melibeus' and Other Vernacular Works of Albertano da Brescia », PhD thesis, Univ. of Stirling, 1985).

12 La référence au Ms. 1185 de L'épitre d'Othéa dans la liste formulée par Van Praet ne présente pas de problème. Comme on sait, ce texte fut peut-être le plus grand succès des œuvres de Christine et il est évident qu'on continuait à le lire au cours du quinzième siècle. Le manuscrit de Louis de Bruges fut copié et illustré dans l'atelier de Loyset Liédet qui y travaillait à partir de 1469. Il est intéressant de noter en passant que la bibliothèque de Philippe le Bon avait cinq copies de ce texte, Édouard IV en avait deux, et il y avait des copies dans les bibliothèques de plusieurs autres bibliophiles de l'entourage du duc Philippe : Antoine de Bourgogne, son fils naturel, Philippe de Clèves, les familles Croy et Lalaing. (G. Mombello, La Tradizione Manoscritta dell'Epistre Othea de Christine de Pizan, Torino, Academia delle Scienze, 1967).

13 La situation est beaucoup moins claire dans le cas du Ms. 585 de la Bibliothèque Nationale. La liste donne l'indication que c'est la traduction du De re militari de l'écrivain romain du quatrième siècle Végèce faite par Jean de Vignay, traducteur de plusieurs œuvres pour la famille royale au XIVe siècle. Cette traduction fut vraisemblablement refaite par Christine. Malheureusement ceux qui parlent de cette œuvre de Christine ne l'on pas lue ou la connaissent seulement par l'imprimé de Vérard (1488) ou la traduction en anglais imprimée par William Caxton (1489). Tous les deux ont modifié un peu le texte original, composé vers 1410, probablement pour la formation militaire du dauphin, Louis de Guyenne. En réalité Christine s'est inspirée de Végèce pour formuler un programme militaire pour le temps contemporain, l'organisation d'une armée avec des chefs qualifiés. La France de cette époque avait besoin des deux. Il n'y avait vraiment pas d'autre source disponible et le texte de Végèce était important à cause de l'idée acceptée que Rome était la source et 
l'inspiration de la chevalerie du moyen âge. Comme dit un historien militaire moderne, l'œuvre de Christine est un bon exemple du retour aux études théoriques de l'art de la guerre, presque inconnues depuis l'époque de Végèce. (R.E. Dupuy et T.N. Dupuy, The Encyclopaedia of Military History from 3500 B.C. to the Present (New York, Harper and Row, 1986, p. 400).

Un aspect assez curieux de ce texte est la présence de deux groupes de manuscrits comprenant des différences assez importantes. Le premier groupe, qui comprend les manuscrits plus ou moins contemporains de la composition de l'œuvre, cite Christine comme auteur et commence par un chapitre adressé à Minerve de qui Christine espère recevoir inspiration et aide. Dans les manuscrits les plus anciens, il y a une miniature qui montre la déesse dans les cabinets d'études de Christine prête à offrir ses conseils.

L'autre groupe, en général de la seconde partie du quinzième siècle, supprime toute référence à Christine, et les références à l'auteur prennent une forme masculine. On se demande si, après la disparition de Christine, on n'eut pas l'idée qu'une femme serait incapable d'écrire un livre tellement utile. Mais ce qu'on ne comprend vraiment pas, c'est que deux des manuscrits qui se trouvent à Bruxelles ont les références à Christine, mais que celui de Louis de Bruges, sorti du même atelier que son exemplaire de l'Épître d'Othéa, appartient au second groupe. Également curieux est le fait que la traduction de Caxton, qui au début de sa carrière avait des liens importants avec Bruges, parle de Christine, bien que l'imprimé Vérard d'à peu près la même époque n'ait pas de référence à l'auteur. On n'arrive pas à identifier la source du manuscrit qui a été copié pour la bibliothèque de Louis de Bruges.

Un autre cas curieux est un manuscrit des Sept Psaumes allégorisés qui a été acheté en 1977 par la Bibliothèque Royale de Bruxelles chez un libraire à Paris. Avant cette date existaient seulement deux manuscrits de cette œuvre et un troisième avait disparu. Christine avait composé ce commentaire sur les sept psaumes de la pénitence en 1409 à la demande de Charles le Bon, roi de Navarre. Dans la paraphrase du psaume 101, on trouve les noms du roi Charles VI de France et de Jean sans Peur, duc de Bourgogne, dans les deux premiers manuscrits (Paris, B.N. nouv. acq. fr. 4792 et Bruxelles, Bibl. Roy. Ms. 10987). Dans le manuscrit récemment trouvé, apparemment copié à Bruges, ces noms sont remplacés par ceux du roi Louis XI et « du bon duc Philippe » et son fils Charles. Il est donc évident que ce manuscrit date des années entre 1461 - quand Louis monta sur le trône de France - et la mort de Philippe le Bon en 1467.

Il y a une signature inversée au fol. 95 de ce manuscrit qui rappelle celle du Harley Ms. 4431. On connaît une telle signature dans seulement un autre manuscrit de cette bibliothèque, ce qui inspire des doutes sur l'authenticité, mais on peut supposer que Louis a fait exécuter ces signatures par un copiste ou secrétaire. Et qui d'autre aurait fait produire un tel manuscrit à Bruges à cette époque?

Le problème le plus curieux de tous, c'est qu'il y a un manuscrit venu de cette bibliothèque qui est apparemment resté inconnu par ceux qui ont étudié la collection. Il ne se trouve pas mentionné dans le livre de Martens. C'est le Ms. fr. 11177 de la Bibliothèque Nationale qui contient La Cité des Dames et Le Livre des Trois Vertus. Ce manuscrit est peut-être un peu moins impressionnant que L'Épitre d'Othéa et Les Faits d'Armes et de Chevalerie, mais les miniatures au début des deux textes sont agréables. Le premier, où les trois vertus apparaissent à Christine dans son cabinet de travail, est assez conventionnel; mais celui au commencement du Livre des Trois Vertus est plutôt exceptionnel, car on voit Christine en train de présenter son livre à Marguerite de 
Guyenne, image suivie par la dédicace à cette princesse, ce qui suggère une origine bourguignonne du manuscrit. Cette dédicace et le fait que Marguerite était la sœur de Philippe le Bon expliquent l'existence de plusieurs manuscrits de ces textes dans les bibliothèques de la famille Croy, de Philippe de Clèves, et peut-être de Jean de Créquy (Ms. de Yale), tous contemporains de Louis de Bruges.

Néanmoins, il n'y a pas de doute que le Ms. 1177 vient de la bibliothèque de Louis de Bruges, car si l'on regarde le premier folio contre une forte lumière on voit bien l'écusson de Louis de Bruges recouvert par celui de Louis XII. Il y a aussi une indication de l'emplacement du volume sur les rayons du château de Blois.

On comprend difficilement pourquoi Van Praett, pendant ses années passées à la Bibliothèque Nationale, n'a jamais remarqué ce manuscrit, qui venait certainement de Bruges. L'évidence de la présence de ce texte à Bruges est confirmée par le fait que c'est dans cette ville que Louis de Baenst, bourgmestre, a commandé une traduction en flamand en 1475 (British Museum, Add. Ms. 20698).

21 Encore un aspect intéressant et important, à propos de La Cité des Dames : ces dernières années, on m'a demandé plusieurs fois si je croyais que Marguerite de Navarre avait lu La Cité des Dames. Je répondais toujours par l'affirmative, car on sait bien qu'une partie importante de l'éducation de cette princesse se faisait dans la bibliothèque du Château de Blois où elle avait tout le loisir de lire les manuscrits venus de Bruges.

Comme j'ai dit en commençant, le but de cette communication est de corriger des erreurs dans le livre récent sur la bibliothèque de Louis de Bruges. Mais en me rappelant la formation de cette bibliothèque, j'ai beaucoup pensé à ces autres bibliothèques formées par ceux qui ont fréquenté la cour de Bourgogne, surtout à l'époque de Philippe le bon. Toutes ces bibliothèques avaient des œuvres de Christine de Pizan. On peut étudier, par exemple, la bibliothèque de plus d'une génération de la famille Croy, à travers des manuscrits qui sont passés à la bibliothèque de Marguerite d'Autriche, qui était aussi bibliophile. On peut ainsi aboutir à une bonne compréhension de leur mentalité aussi bien qu'à une connaissance de leurs intérêts. La personnalité de Louis de Bruges, bien entendu, est assez loin de celle de Marguerite d'Autriche, mais à travers l'étude de leurs manuscrits, on peut arriver à une meilleure approche de la qualité de leurs vies.

\section{NOTES}

1. Texte d'une communication donnée au deuxième colloque international sur Christine de Pizan, Orléans, juillet 1995, n'ayant pu paraître dans les Actes. 